\title{
Orexin-A alleviates cerebral ischemia-reperfusion injury by inhibiting endoplasmic reticulum stress-mediated apoptosis
}

\author{
DANDAN XU ${ }^{1 *}$, TINGTING KONG ${ }^{1 *}$, BAOHUA CHENG $^{2}$, RUMIN ZHANG $^{2}$, \\ CHUNQING YANG ${ }^{2}$, JING CHEN ${ }^{2,3}$ and CHUNMEI WANG ${ }^{2}$ \\ ${ }^{1}$ Cheeloo College of Medicine, Shandong University, Jinan, Shandong $250014 ;{ }^{2}$ Neurobiology Key Laboratory of \\ Jining Medical University in Colleges of Shandong, Jining, Shandong 272067, P.R. China; \\ ${ }^{3}$ Division of Biomedical Sciences, Warwick Medical School, University of Warwick, Coventry CV4 7AL, UK
}

Received March 27, 2020; Accepted January 8, 2021

DOI: $10.3892 / \mathrm{mmr} .2021 .11905$

\begin{abstract}
Orexin-A (OXA) protects neurons against cerebral ischemia-reperfusion injury (CIRI). Endoplasmic reticulum stress (ERS) induces apoptosis after CIRI by activating caspase-12 and the CHOP pathway. The present study aimed to determine whether OXA mitigates CIRI by inhibiting ERS-induced neuronal apoptosis. A model of CIRI was established, in which rats were subjected to middle cerebral artery occlusion with ischemic intervention for $2 \mathrm{~h}$, followed by reperfusion for $24 \mathrm{~h}$. Neurological deficit examination and 2,3,5-triphenyltetrazolium chloride staining were performed to assess the level of CIRI and neuroprotection by OXA. Expression levels of ERS-related proteins and cleaved caspase-3 were measured via western blotting, while the rate of neuronal apoptosis in the cortex was determined using a TUNEL assay. OXA treatment decreased the infarct volume of rats after CIRI and attenuated neuron apoptosis. Furthermore, administration of OXA decreased the expression levels of GRP78, phosphorylated (p)-PERK, p-eukaryotic initiation factor- $2 \alpha, \mathrm{p}$-inositol requiring enzyme $1 \alpha, \mathrm{p}-\mathrm{JNK}$, cleaved caspase-12, CHOP and cleaved caspase-3, all of which were induced by CIRI. Collectively, these findings suggested that OXA attenuated CIRI by inhibiting ERS-mediated apoptosis, thus clarifying the mechanism underlying its neuroprotective
\end{abstract}

Correspondence to: Dr Jing Chen, Division of Biomedical Sciences, Warwick Medical School, University of Warwick, Gibbet Hill Road, Coventry CV4 7AL, UK

E-mail: jing.chen@warwick.ac.uk

Professor Chunmei Wang, Neurobiology Key Laboratory of Jining Medical University in Colleges of Shandong, 133 Hehua Road, Jining, Shandong 272067, P.R. China

E-mail: wangchunmei410@mail.jnmc.edu.cn

*Contributed equally

Key words: orexin-A, cerebral ischemia-reperfusion injury, endoplasmic reticulum stress, apoptosis, ischemic stroke effect and providing a novel therapeutic direction for the treatment of CIRI.

\section{Introduction}

The incidence of stroke around the world is high and continues to increase. Between 1988 and 2010, the incidence of stroke in men in Tianjin, China increased from 136.8/100,000 to $387.0 / 100,000$ and the incidence in women increased from $65.9 / 100,000$ to $249.7 / 100,000$ (1). In the United States $>795,000$ stroke cases occur each year and ischemic stroke accounts for $87 \%$ of all strokes (2). Ischemic stroke, the most common type of stroke, is usually caused by a sudden interruption of blood flow due to a thrombosis or embolism blocking a blood vessel, resulting in brain dysfunction (3). Hence, the most effective method to treat cerebral ischemic stroke is to restore blood flow quickly and completely (4). However, the restoration of blood flow inevitably causes a series of pathophysiological changes, including oxidative stress, mitochondrial dysfunction, excitatory amino acid toxicity, inflammation and infarct formation (5). This process, known as 'cerebral ischemia-reperfusion injury' (CIRI), remains to be fully understood at a mechanistic level (6).

Endoplasmic reticulum stress (ERS) is involved in CIRI, which can be alleviated by inhibiting ERS-induced apoptosis $(7,8)$. As the main site responsible for protein folding and secretion, the endoplasmic reticulum (ER) serves a vital role in maintaining homeostasis within the cellular microenvironment $(9,10)$. In some pathological states, such as ischemia and hypoxia, the homeostasis of the ER is disrupted, causing large quantities of unfolded or misfolded proteins to accumulate in the ER, further exacerbating ERS $(9,10)$. ERS can be maintained within certain limits via the unfolded protein reaction (UPR), but an excessive or long-term activation of the UPR causes ERS-related apoptosis $(9,10)$. ERS causes the molecular chaperone GRP78 to separate from three membrane proteins, PERK, inositol requiring enzyme $1 \alpha$ (IRE1 $\alpha$ ) and activating transcription factor 6 (ATF6), which then activate a series of downstream signal responses (11-14). When the stimulus is intense or persists for a long time, these responses eventually lead to apoptosis. Phosphorylated (p)-JNK, CHOP and caspase-12 are activated by IRE1 $\alpha$ (11-14). Neuronal 
apoptosis is a major pathophysiological change associated with CIRI (15), and attenuating cell death is an important strategy for alleviating permanent damage.

Orexin-A (OXA), a neuropeptide that is primarily secreted by orexin-containing neurons located in the lateral hypothalamus, exerts a neuroprotective effect against CIRI (16). Our previous study initially confirmed that OXA can prevent CIRI in Sprague-Dawley (SD) rats subjected to middle cerebral artery occlusion (MCAO) model and reperfusion (17). In an oxygen/glucose deprivation and reoxygenation model in vitro, it was also revealed that OXA exerts its protective role by inhibiting ERS-mediated apoptosis (18). In the present study, an MCAO model was established to simulate CIRI and investigated whether OXA can exert neuroprotective effects by inhibiting ERS-mediated apoptosis in vivo. The present findings will help clarify the mechanism underlying the neuroprotective effect of OXA and provide an experimental basis for the treatment of ischemic stroke.

\section{Materials and methods}

Animals. A total of 90 male SD rats (age, 8-9 weeks; weight, $250 \pm 10 \mathrm{~g}$ ) were purchased from Jinan Pengyue Experimental Animal Breeding, Co., Ltd. All animals were given water and food ad libitum, and were maintained on a 12-h light/dark cycle in a temperature-controlled room at $24-26^{\circ} \mathrm{C}$ with humidity of 50-65\%. All rats acclimated for 1 week before experimental procedures. All animal experiments were approved by the Animal Ethics Committee of Jining Medical University, and performed were in accordance with the National Experimental Animal Feeding Guidelines (19). The rats were sacrificed with decapitation after deep anesthesia causing rapid and unconscious death without pain.

MCAO model. SD rats were fasted for $12 \mathrm{~h}$ before the experiment. Rats were anesthetized using $10 \%$ chloral hydrate (300 $\mathrm{mg} / \mathrm{kg}$ ) via intraperitoneal injection for up to $2 \mathrm{~h}$ after reperfusion. After intraperitoneal injection of $10 \%$ chloral hydrate, the rats did not show symptoms of peritonitis. After a midline incision was introduced in the neck, the right common carotid artery, right external carotid artery and internal carotid artery (ICA) were exposed and separated. A 2.5 nylon mono-filament $(0.265 \mathrm{~mm}$ in diameter) was inserted through the common carotid artery into the lumen of the ICA and advanced 18-22 $\mathrm{mm}$ from the bifurcation until it blocked the origin of the right middle cerebral artery. In the drug and model groups, the filament remained in the lumen for $2 \mathrm{~h}$, and was subsequently withdrawn to allow reperfusion for $3,6,12$, 24 and $48 \mathrm{~h}$. The sham-operated group was treated the same as the model group except for the occlusion of the middle cerebral arteries after the neck incision. A total of 30 rats were randomly divided into 6 groups: sham operation group (Sham), MCAO model group and reperfusion for $3 \mathrm{~h}$ (CIRI $3 \mathrm{~h}), 6 \mathrm{~h}$ (CIRI 6h), 12 h (CIRI 12h), 24 h (CIRI 24h) and 48 h (CIRI 48h). Based on the expression of ERS-related proteins, CIRI 24h was selected for OXA intervention. Therefore, 20 rats were randomly divided into the sham operation group (Sham), the MCAO model group and reperfusion for $24 \mathrm{~h}$ (CIRI), CIRI with intracerebroventricular (ICV) injection of normal saline (NS; CIRI + NS) and CIRI with intracerebroventricular (ICV) injection of OXA (CIRI + OXA). Another 40 rats were used for TTC and TUNEL staining. In addition, there was $\sim 1 / 3$ mortality and model failure rate.

Intracerebroventricular (ICV) injection. At the beginning of reperfusion, the rats were anesthetized via intraperitoneal injection with $10 \%$ chloral hydrate $(300 \mathrm{mg} / \mathrm{kg})$. A burr hole for ICV administration was carefully made in the skull at $0.8-\mathrm{mm}$ dorsal and 1.6- $\mathrm{mm}$ lateral to the right from Bregma using a Dremel drill. A total of $10 \mu \mathrm{l}$ OXA (30 $\mu \mathrm{g} / \mathrm{kg})(20)$ from Phoenix Pharmaceuticals, Inc. in $0.9 \% \mathrm{NaCl}$ or $10 \mu \mathrm{NS}$ $(0.9 \% \mathrm{NaCl})$ were injected at $2 \mu \mathrm{l} / \mathrm{min}$ for $5 \mathrm{~min}$.

Neurological score. After reperfusion for $24 \mathrm{~h}$, neurological function of all rats was evaluated according to the Longa five-point scale (21): 0 , no neurological deficit; 1 , failed to fully extend their left forepaw; 2, circling to the left when walking; 3 , falling to the left when walking; and 4, failure to walk spontaneously or stroke-related mortality. Only rats scoring 1, 2 or 3 were selected for use in subsequent experiments. The test was performed in a blinded manner.

2,3,5-Triphenyl-2H-tetrazolium chloride (TTC) staining. Rats were anesthetized with $10 \%$ chloral hydrate $(300 \mathrm{mg} / \mathrm{kg})$, and brains were collected and incubated on ice for $30 \mathrm{~min}$. Coronal thin slices $(2 \mathrm{~mm})$ of brain were stained with $1 \%$ TTC (Sigma-Aldrich; Merck KGaA) and incubated at $37^{\circ} \mathrm{C}$ in the dark for 15-20 min. Viable tissues were stained deep red, whereas infarcts were pale white. TTC-stained brains were then fixed with $4 \%$ formaldehyde for $24 \mathrm{~h}$ at room temperature and imaged with a digital camera. The infarct areas were measured using the ImageJ 2 software (National Institutes of Health).

TUNEL assay. TUNEL staining was performed according to the manufacturer's instructions (Promega Corporation). Briefly, coronal slices $(30 \mu \mathrm{m})$ were fixed with $4 \%$ paraformaldehyde for $30 \mathrm{~min}$ at room temperature and permeabilized with $0.3 \%$ Triton $\mathrm{X}-100$ for $10 \mathrm{~min}$ at room temperature. After equilibration for $10 \mathrm{~min}$, the slices were incubated with rTdT reaction buffer for $60 \mathrm{~min}$ at a constant temperature of $37^{\circ} \mathrm{C}$ and then incubated with $2 \mathrm{X} \mathrm{SSC}$ for $15 \mathrm{~min}$ to stop the rTdT reaction at a constant temperature of $37^{\circ} \mathrm{C}$. Finally, the nuclei were counterstained with DAPI $(100 \mathrm{ng} / \mathrm{ml})$ for $30 \mathrm{~min}$ at room temperature, and then the anti-fluorescence quencher was added dropwise for mounting. Images of three fields of view were randomly capture under an optical microscope with a magnification of x200 (Olympus IX 71; Olympus Corporation) and the percentages of TUNEL-positive cells vs. total cells were calculated

Western blot analysis. Brain tissues of rats were homogenized in RIPA lysis buffer (Beyotime Institute of Biotechnology) supplemented with PMSF (Beyotime Institute of Biotechnology), and protein concentrations were quantified using the BCA method (Beyotime Institute of Biotechnology). Equal amounts of protein $(25 \mu \mathrm{g})$ from each sample were separated via $10 \%$ SDS-PAGE and transferred to PVDF membranes at $4^{\circ} \mathrm{C}$. The membranes were blocked with $5 \%$ non-fat milk powder in TBS-Tween-20 $(0.1 \%)$ for $2 \mathrm{~h}$ at room temperature and then 

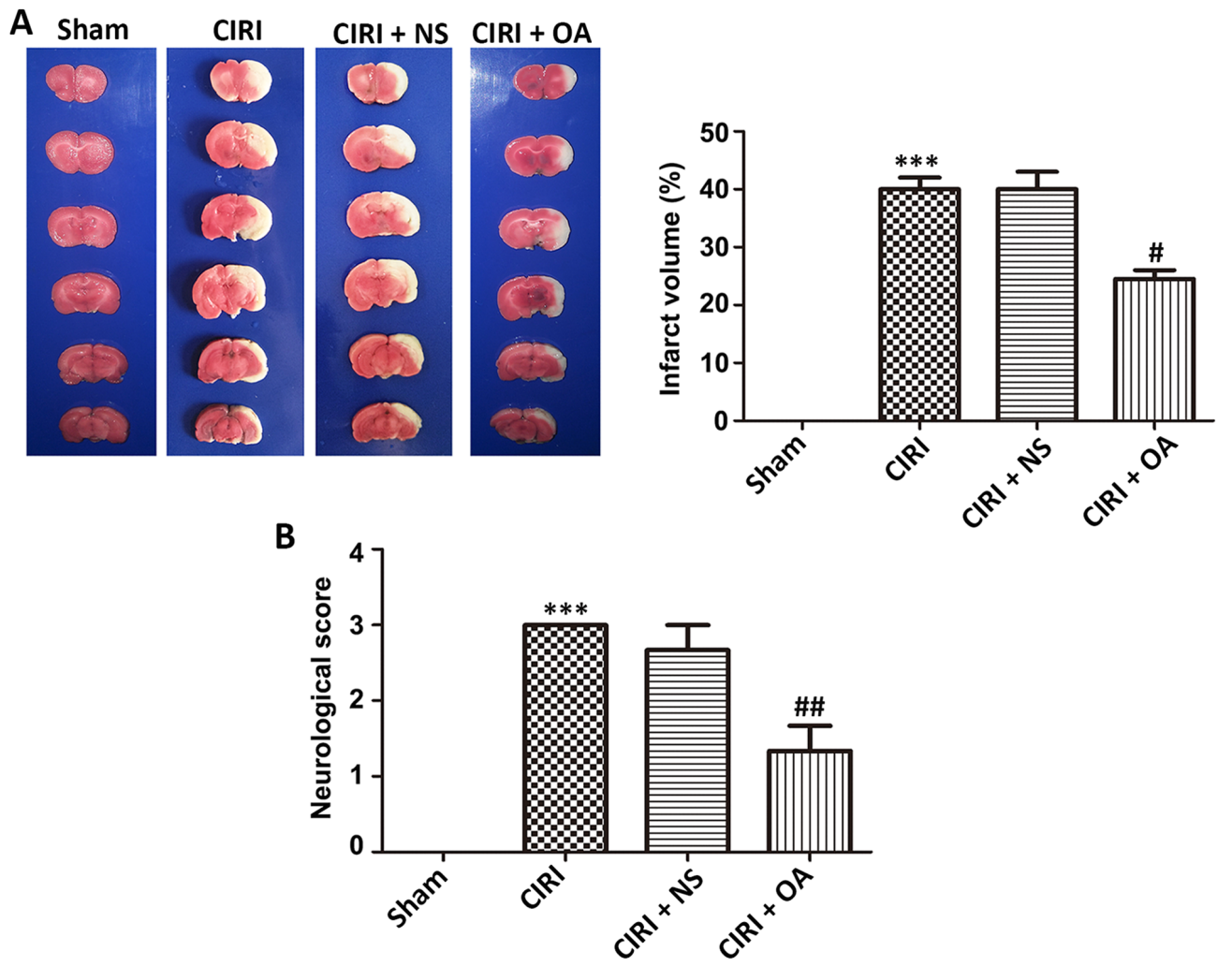

Figure 1. Effect of OXA on cerebral infarct volume and neurological deficit score caused by CIR. (A) Representative TTC-stained coronal brain sections and quantification of infarct volume. (B) Neurological deficit score. Data are presented as mean \pm SEM $(n=3) .{ }^{* * * *} \mathrm{P}<0.001$ vs. Sham group; ${ }^{*} \mathrm{P}<0.05$ and ${ }^{\# \#} \mathrm{P}<0.01$ vs. CIRI group. CIRI, cerebral ischemia-reperfusion injury; NS, normal saline (0.9\% NaCl); OA, Orexin-A.

incubated overnight at $4^{\circ} \mathrm{C}$ with the following primary antibodies: Anti-cleaved caspase-3 (cat. no. 9661S), anti-GRP78

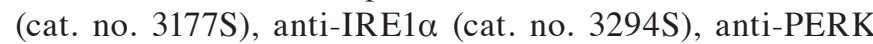
(cat. no. 5683S), anti-p-PERK (cat. no. 3179S), anti-CHOP (cat. no. 2895S), anti-eIF2 $\alpha$ (cat. no. 5324S), anti-p-eIF2 $\alpha$ (cat. no. 9721S), anti-JNK (cat. no. 9252T), anti-p-JNK (cat. no. $9255 \mathrm{~S}$ ), anti-cleaved caspase-12 (cat. no. 35965S) (all 1:1,000; Cell Signaling Technology, Inc.), anti-p-IRE1 $\alpha$ (cat. no. ab48187; $1: 1000 ;$ Abcam) and anti- $\beta$-actin (cat. no. TA-09; 1:1,000; OriGene Technologies, Inc.). Next, the membranes were incubated for $1 \mathrm{~h}$ at room temperature with horseradish peroxidase-conjugated anti-rabbit IgG or anti-mouse IgG secondary antibodies (1:5,000; OriGene Technologies, Inc.), and visualized using an ECL system (MultiSciences Biotech Co,. Ltd. The grayscale intensities of protein bands were quantified using ImageJ 2 software (National Institutes of Health).

Statistical analysis. All data are representative of $\geq 3$ independent experiments and were analyzed using the GraphPad Prism 5 software (GraphPad Software, Inc.). Data are presented as the mean \pm SEM. One-way ANOVA followed by Tukey's test were performed. $\mathrm{P}<0.05$ was considered to indicate a statistically significant difference.

\section{Results}

OXA decreases infarct volume and improves neurological deficit score in brain damage caused by CIRI. To evaluate the neuroprotective effect of OXA in the CIRI model, infarct volume and neurological deficit score were measured, respectively.
Healthy cerebral tissues were stained red, whereas infarcted areas were stained white (Fig. 1A). No infarction areas were detected in the sham group, whereas the CIRI group had a significantly higher rate of infarction $(40 \pm 2 \%)$ compared with the sham group $(\mathrm{P}<0.001)$, demonstrating that CIRI-induced cerebral infarct had been successfully introduced. However, the OXA group had a significantly lower rate of infarction $(24.5 \pm 1.5 \%)$ compared with the CIRI group $(\mathrm{P}<0.05)$, demonstrating that intervention with OXA significantly decreased the infarct area.

As presented in Fig. 1B, the average neurological deficit scores of the CIRI model rats was 3 compared with the sham group, according to the Longa score scale $(\mathrm{P}<0.001)$. After OXA intervention, neurological deficit scores were significantly decreased $(\mathrm{P}<0.01)$, indicating that OXA has the potential ability to improve neurological deficit scores. Thus, OXA exhibited a notable neuroprotective effect on infarct volume and neurological deficit score in CIRI model rats.

Expression levels of ERS-related proteins are elevated in the cortex after CIRI. To determine whether CIRI was accompanied by ERS, western blot analysis was conducted to evaluate the expression levels of ERS-related proteins in the cerebral cortex on the ischemic side at different times post-reperfusion. The expression patterns of five proteins exhibited significant differences at various times during the post-reperfusion period (Fig. 2). After IR, the expression level of HSPA5 was increased to a peak at $6 \mathrm{~h}$ and then declined $(\mathrm{P}<0.01$ and $\mathrm{P}<0.001)$. The phosphorylation level of PERK was elevated at 3 and $6 \mathrm{~h}$, then decreased before increasing again from 

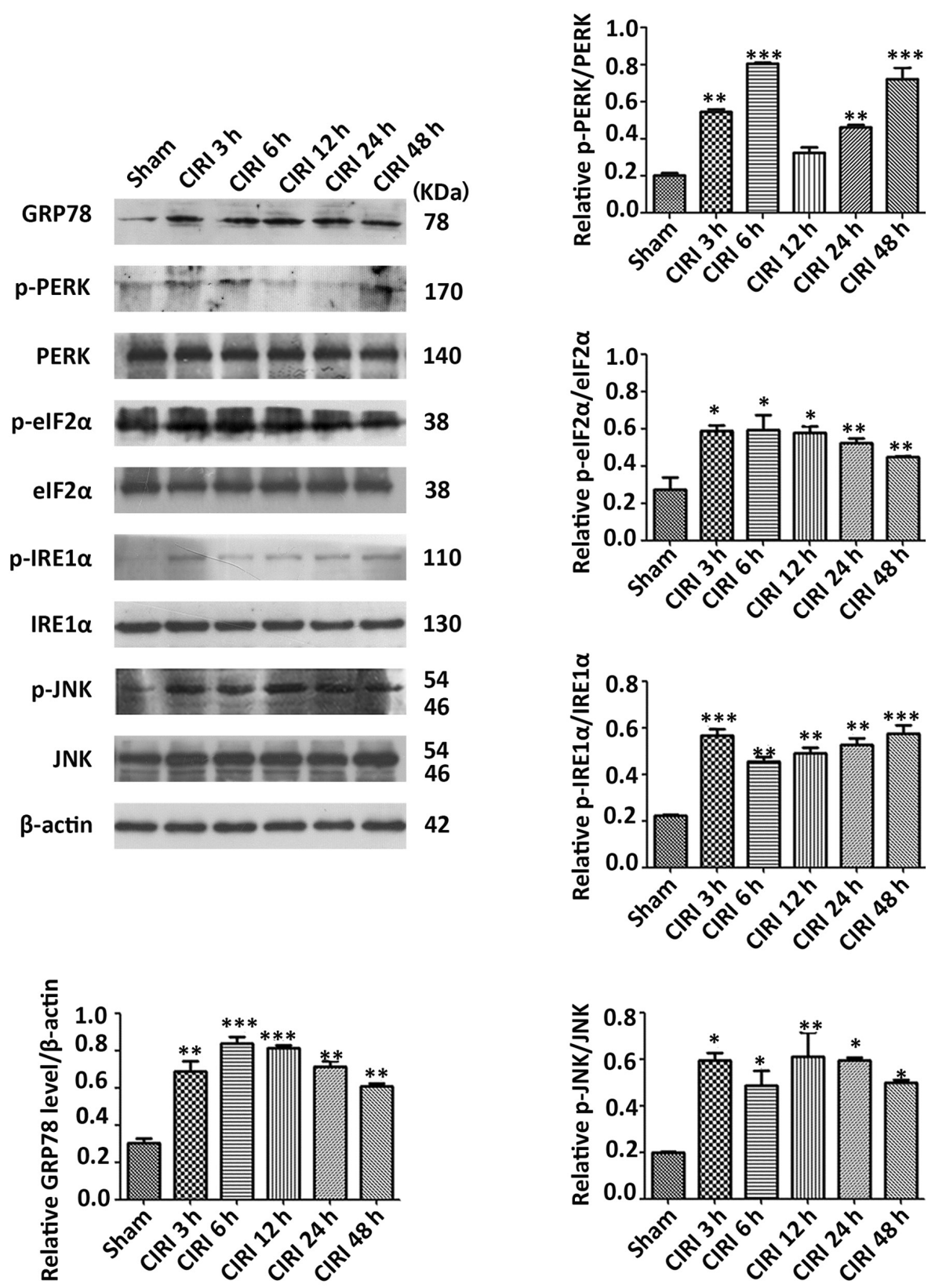

Figure 2. Expression levels of ERS-related proteins induced by cerebral ischemia-reperfusion. Western blot analysis results of ERS-related proteins in cortex of rats after the indicated durations of CIRI. Semi-quantitative analysis is presented for HSPA5, p-PERK, PERK, p-eIF2 $\alpha$, eIF2 $\alpha$, p-IRE1 $\alpha$, IRE1 $\alpha$, p-JNK and JNK. Data are presented as mean \pm SEM $(n=3) .{ }^{*} \mathrm{P}<0.05,{ }^{* *} \mathrm{P}<0.01$ and ${ }^{* * * *} \mathrm{P}<0.001$ vs. Sham group. CIRI, cerebral ischemia-reperfusion injury; $\mathrm{p}-$, phosphorylated; HSPA5, heat shock protein family A (Hsp70) member 5; eIF2 $\alpha$, eukaryotic translation initiation factor 2; IRE1 $\alpha$, inositol requiring enzyme $1 \alpha$.

$24 \mathrm{~h}(\mathrm{P}<0.01$ and $\mathrm{P}<0.001)$. The expression of $\mathrm{p}$-eIF $2 \alpha$ was highest at $6 \mathrm{~h}$ post-reperfusion, and then gradually decreased $(\mathrm{P}<0.05$ and $\mathrm{P}<0.01) . \mathrm{p}-\mathrm{IRE} 1 \alpha$ reached its highest levels at $3 \mathrm{~h}$ post-reperfusion, and then decreased $(\mathrm{P}<0.01$ and $\mathrm{P}<0.001)$. The expression of $\mathrm{p}-\mathrm{JNK}$ remained at a high level for $48 \mathrm{~h}$ post-reperfusion $(\mathrm{P}<0.05$ and $\mathrm{P}<0.01)$. These observations demonstrate that the patterns of gene expression are complex and diverse, increasing the challenge of understanding the molecular pathology of CIRI. Taken together, however, the present findings suggested that these proteins were activated in CIRI, indicating that ERS is accompanied by CIRI.

OXA significantly decreases the expression of ERS-related protein in the cortex caused by CIRI. To investigate the effect of OXA on the expression of ERS-related proteins, CIR was simulated by $2 \mathrm{~h}$ MCAO followed by $24 \mathrm{~h}$ of reperfusion, and OXA was administered via ICV injection during reperfusion. Next, the expression levels of ERS-related protein were detected via western blotting (Fig. 3A). Compared with Sham group, the fold changes of the expression levels of HSPA5, p-PERK, p-eIF2 $\alpha, p$-IRE1 $\alpha$, and p-JNK in CIRI group and CIRI + OA group were uneven. GRP78 expression was significantly higher in the CIRI group (22.6-fold) compared with the Sham model (Fig. 3B), but significantly lower in the OXA model compared with the CIRI group $(\mathrm{P}<0.01)$. No statistically significant difference was observed between CIRI and CIRI + NS models. The phosphorylation levels of PERK, eIF2 $\alpha$, IRE1 $\alpha$ and JNK were upregulated in the CIRI group compared with the Sham group $(\mathrm{P}<0.05, \mathrm{P}<0.01$ and $\mathrm{P}<0.001)$, but all were downregulated following intervention 
A

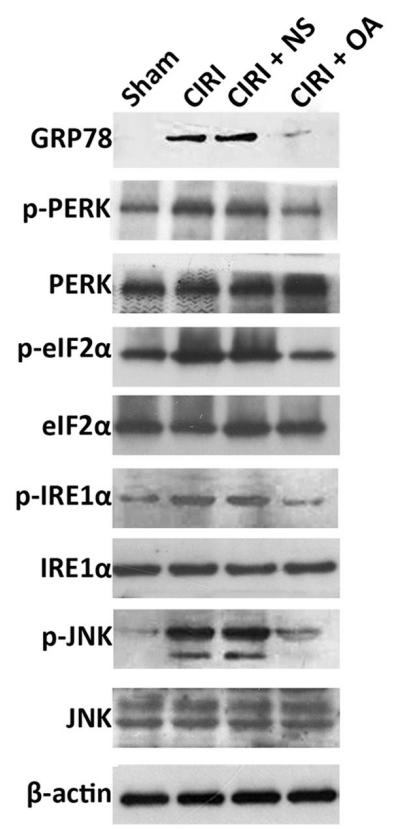

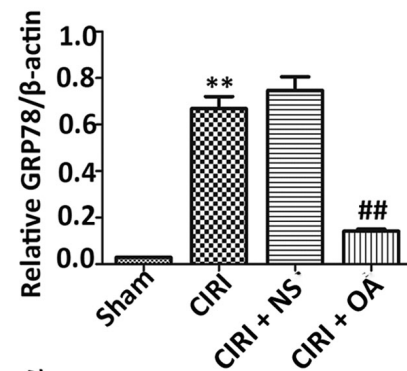
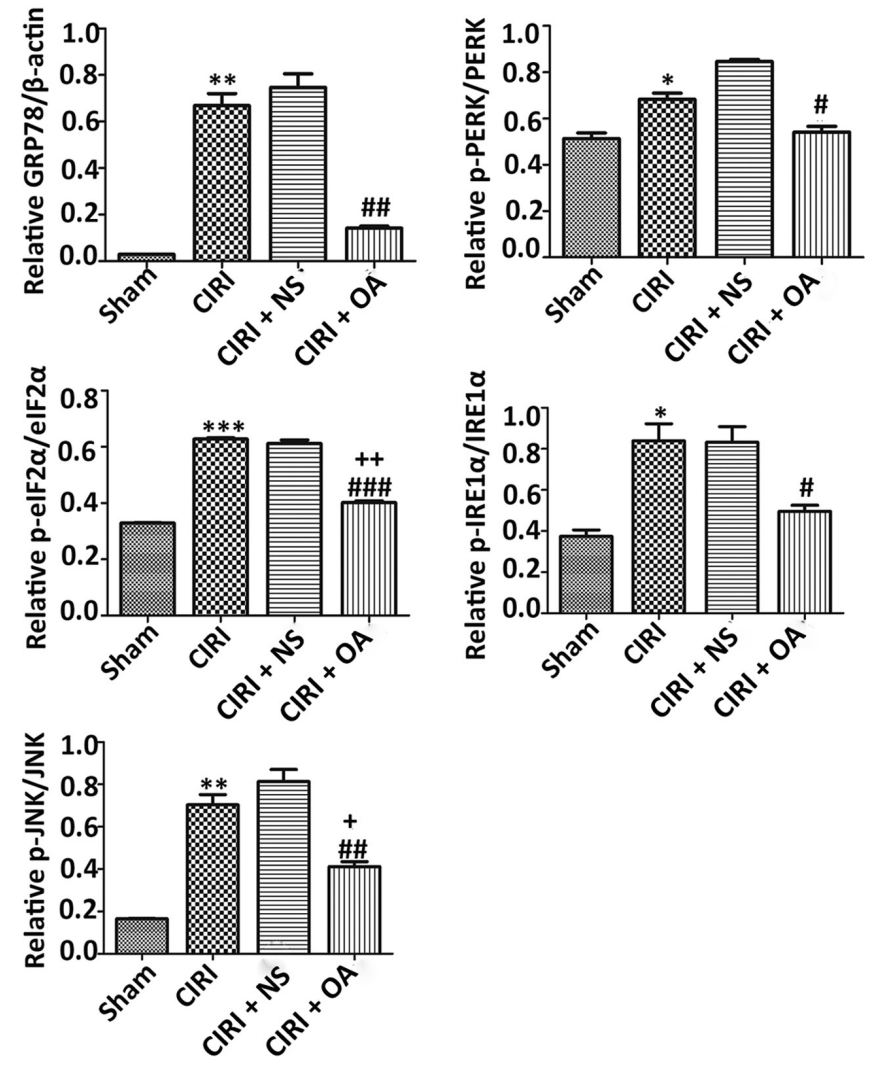

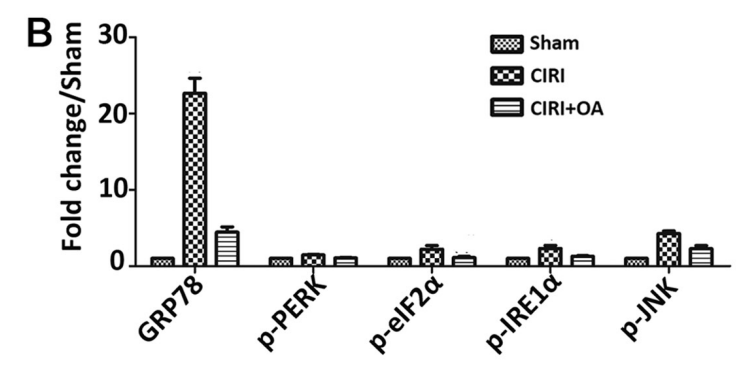

Figure 3. Effect of OXA on expression levels of ERS-related proteins induced by CIR. (A) Western blotting and semi-quantitative analyses of HSPA5, p-PERK, PERK, p-eIF2 $\alpha$, eIF2 $\alpha$, p-IRE1 $\alpha$, IRE1 $\alpha$, p-JNK and JNK. Treatment with OXA significantly decreased the expression levels of HSPA5, p-PERK, p-eIF2 $\alpha$, p-IRE1 $\alpha$ and p-JNK. (B) Compared with Sham group, the fold changes of the expression levels of HSPA5, p-PERK, p-eIF2 $\alpha, p-I R E 1 \alpha$ and p-JNK in CIRI group and CIRI + OA group were uneven. Data are presented as mean $\pm \mathrm{SEM}(\mathrm{n}=3)$. ${ }^{*} \mathrm{P}<0.05,{ }^{* *} \mathrm{P}<0.01$ and ${ }^{* * *} \mathrm{P}<0.001$ vs. Sham group; ${ }^{*} \mathrm{P}<0.05$, ${ }^{\# \#} \mathrm{P}<0.01$ and ${ }^{\# \# \#+P} \mathrm{P}<0.001$ vs. CIRI group; ${ }^{\mathrm{P}} \mathrm{P}<0.05$ and ${ }^{++} \mathrm{P}<0.01$ vs. sham group. CIRI, cerebral ischemia-reperfusion injury; $\mathrm{NS}$, normal saline ( $\left.0.9 \% \mathrm{NaCl}\right) ; \mathrm{OA}, \mathrm{Orexin}-\mathrm{A} ; \mathrm{p}-$, phosphorylated; HSPA5, heat shock protein family A (Hsp70) member 5; eIF2 $\alpha$, eukaryotic translation initiation factor 2; IRE1 $\alpha$, inositol requiring enzyme 1 $\alpha$.

with OXA $(\mathrm{P}<0.05, \mathrm{P}<0.01$ and $\mathrm{P}<0.001)$. In addition, only the phosphorylation levels of eIF $2 \alpha$ and JNK were statistically significant between the Sham and OXA models $(\mathrm{P}<0.05$ and $\mathrm{P}<0.01)$. In brief, these results indicated that CIRI activated ERS-related proteins in the rat cortex, and that OXA could decrease their activities.

OXA significantly decreases the expression levels of CHOP, cleaved caspase-12 and cleaved caspase-3 in the cortex following CIRI. The caspase-12/caspase-9/caspase-3 or CHOP apoptotic pathways are mediated by ERS (22). Moreover, caspase-12 is regarded as a representative molecule in ERS-mediated apoptosis, and caspase-3 serves a key role in regulating apoptosis, which can directly lead to cell death $(22,23)$. Therefore, the expression levels of CHOP, cleaved caspase-12 and cleaved caspase-3 were measured using western blotting (Fig. 4). CIRI upregulated the expression of all three proteins (all $\mathrm{P}<0.05$ ). Moreover, treatment with OXA significantly decreased the expression of CIRI-induced CHOP, cleaved caspase-12 and cleaved caspase-3 (all $\mathrm{P}<0.05$ ). However, the expression levels of these proteins did not differ significantly between the CIRI + NS and CIRI groups. These data suggested that OXA exerted an anti-apoptotic effect on CIRI by inhibiting the expression of apoptosis-related genes.

OXA significantly decreases apoptosis in CIRI models. To determine the effect of OXA on apoptosis in the CIRI model, apoptotic cells in brain tissues were detected using TUNEL staining. TUNEL-positive cells were barely visible in the Sham group, whereas substantial levels of TUNEL-positive neurons (47.33\%) were detected in the CIRI group (Fig. 5). However, the number of TUNEL-positive cells in the CIRI + OXA group was significantly decreased $(28.33 \%)$ compared with the CIRI group $(\mathrm{P}<0.01)$. These results 
A

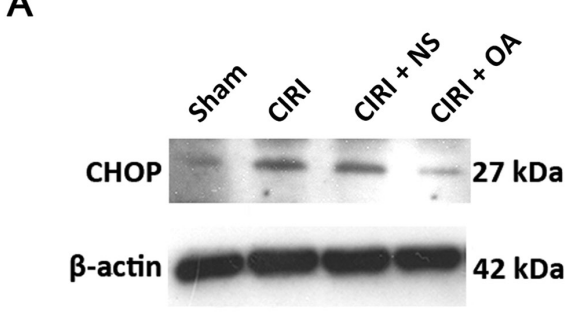

B

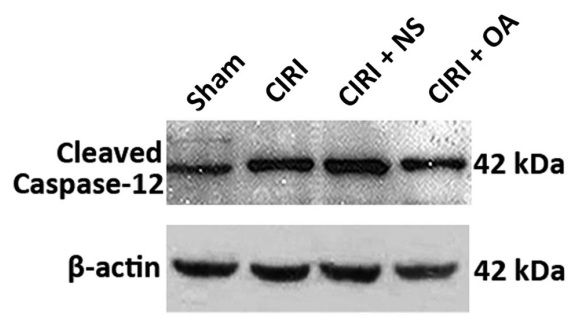

C

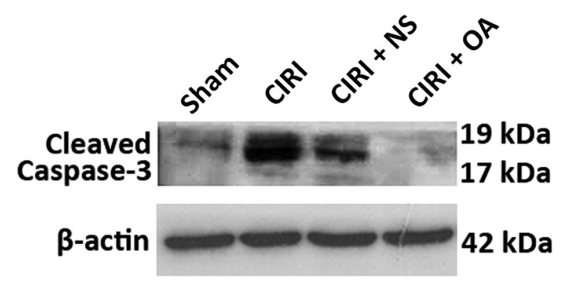

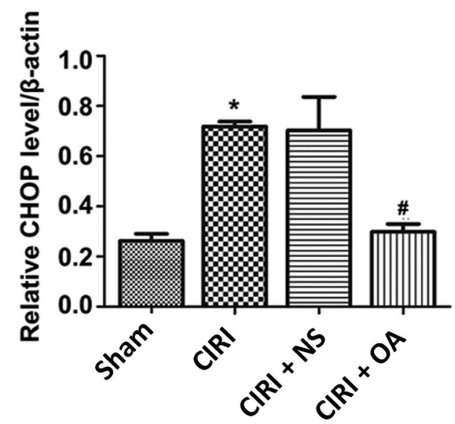
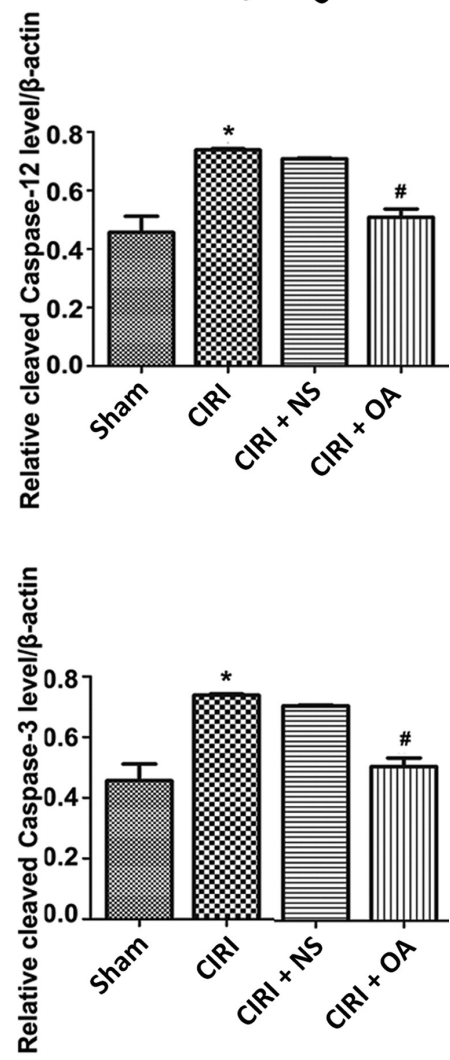

Figure 4. Effect of OXA on the expression levels of CHOP, cleaved caspase-12 and cleaved caspase-3 following CIR. Western blotting and semi-quantitative analyses of (A) CHOP, (B) cleaved caspase-12 and (C) cleaved caspase-3. Treatment with OXA significantly decreased the expression levels of CHOP, cleaved caspase-12 and cleaved caspase-3. Data are presented as mean $\pm \operatorname{SEM}(n=3)$. ${ }^{*} \mathrm{P}<0.05$ vs. Sham group; ${ }^{*} \mathrm{P}<0.05$ vs. CIRI group. CIRI, cerebral ischemia-reperfusion injury; NS, normal saline $(0.9 \% \mathrm{NaCl})$; OA, Orexin-A.

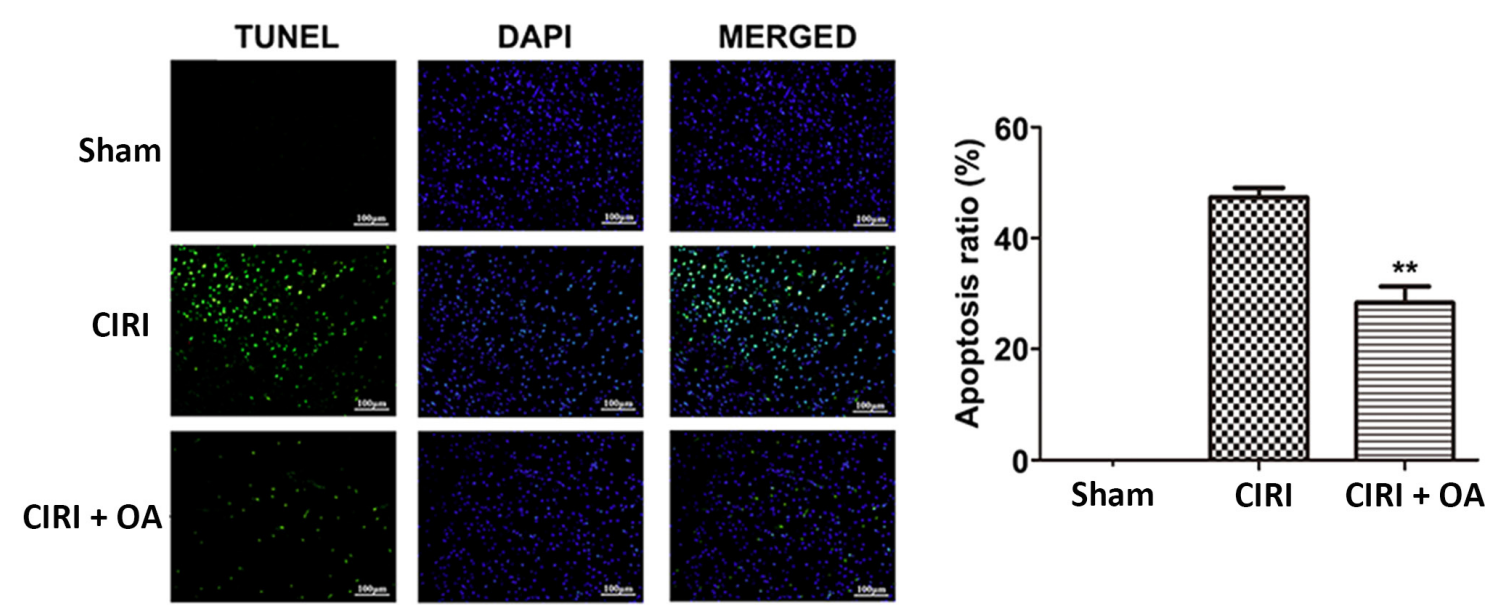

Figure 5. Effect of OXA on apoptosis in the cortex following CIR. TUNEL staining of brain tissue slices. Nuclei were counterstained with DAPI. TUNEL and DAPI images were merged. TUNEL-positive frequency was statistically analyzed. Scale bar, $100 \mu \mathrm{m}$. Data are presented as mean \pm SEM ( $\mathrm{n}=3$ ). ${ }^{* *} \mathrm{P}<0.01$ vs. CIRI group. CIRI, cerebral ischemia-reperfusion injury; OA, Orexin-A. 


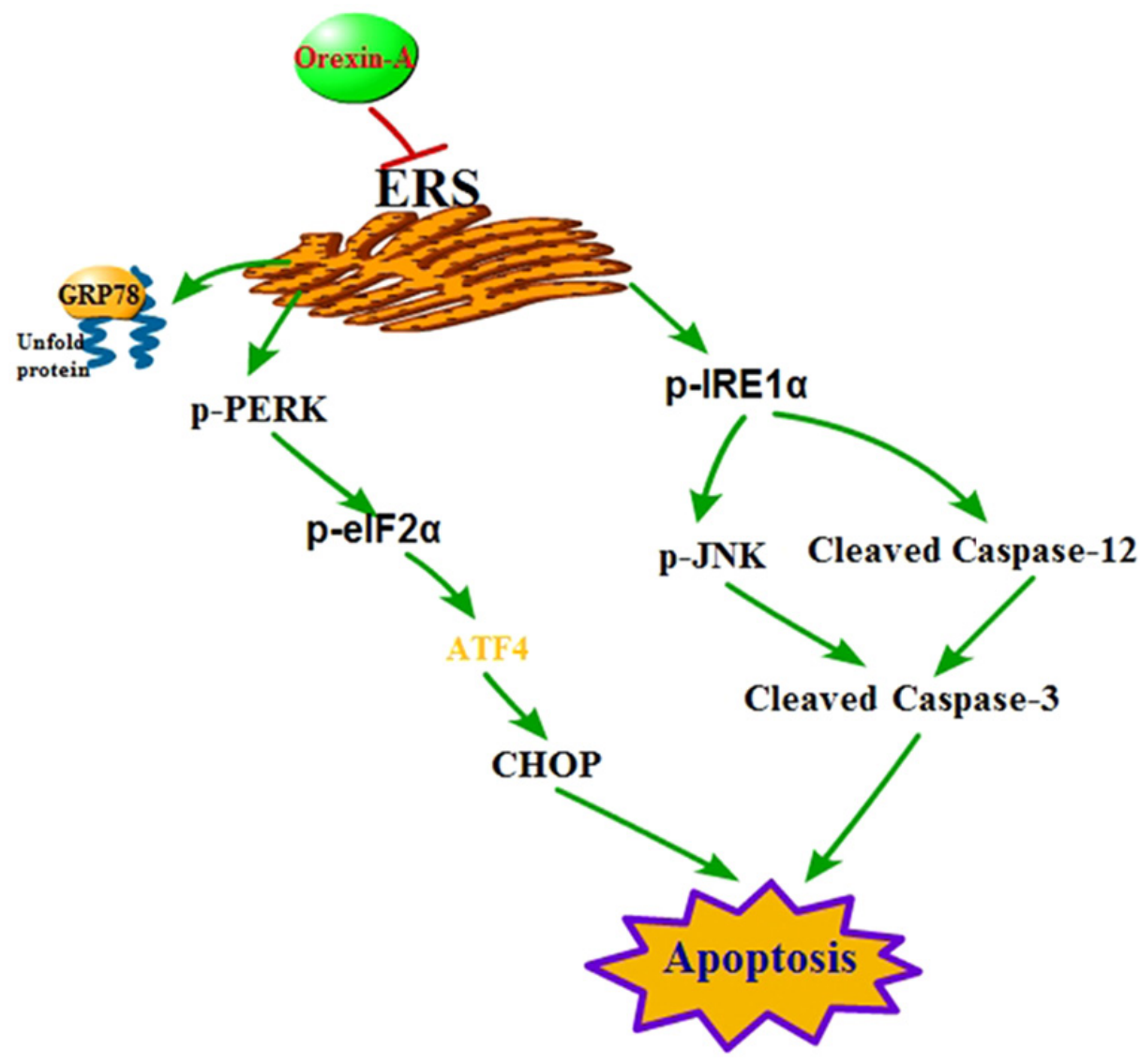

Figure 6. Proposed mechanism via which OXA attenuates CIRI. Prolonged or excessive ERS induced by CIRI separates HSPA5 from IRE1 $\alpha$ and PERK, and activates two apoptosis signaling pathways of UPR: IRE1 $\alpha$ pathway and PERK pathway. OXA can inhibit apoptosis by regulating the expression of CHOP via PERK/eIF2 $\alpha$ pathway, and the expression levels of JNK and caspase-12 via IRE1 $\alpha$ pathway. ERS, endoplasmic reticulum stress; p-, phosphorylated; eIF2 $\alpha$, eukaryotic translation initiation factor 2; IRE1 $\alpha$, inositol requiring enzyme 1 $\alpha$; ATF4, activating transcription factor 4.

demonstrated that OXA exerted an anti-apoptotic effect in the cortex following CIR.

\section{Discussion}

Previous research established the neuroprotective effect of OXA on CIRI (16), but the underlying mechanism remained unknown. Moreover, our previous study demonstrated that the neuroprotective effect of OXA was achieved by inhibiting ERS-mediated apoptosis in vitro (18). The present study demonstrated that, in rats, OXA protected the brain against CIRI by attenuating ERS-mediated apoptosis.

The prevention and treatment of CIRI have gained increased attention in the field of global IR research $(24,25)$. Blocking neuronal apoptosis is the most important step in alleviating CIRI (26). Apoptosis is a major mode of neuron death following CIRI, particular apoptosis induced by the ERS pathway (8). ER is the main site of protein synthesis, processing and transport. Accordingly, the ERS response can be induced by a series of pathophysiological changes that occur in CIRI, such as $\mathrm{Ca}^{2+}$ overload, oxidative stress, metabolic disorders and inflammatory responses, all of which disrupt the homeostasis of the ER (5-7). The present results study confirm these effects. In the current model, CIRI was induced in rats by MCAO for $2 \mathrm{~h}$, followed reperfusion for various times. Time-dependent changes in the expression of molecular markers of ERS, including HSPA5, p-PERK,
p-eIF $2 \alpha, p-I R E 1 \alpha$ and p-JNK, indicated that ERS serves a critical role in brain damage after CIR.

The ERS response promotes the processing of misfolded or unfolded proteins accumulated in the ER, which helps to maintain the physiological function of cells, but excessively long or strong ERS can cause apoptosis $(9,10)$. A type of ERS response, the UPR, has been studied extensively, and the mechanisms have been described. The signal transduction pathways of UPR are 3-fold, comprising the PERK, ATF6 and IRE1 $\alpha$ pathways. In a stress-free state, these three transmembrane proteins are restrained by binding to HSPA5. HSPA5 itself is a well-established hallmark of ERS, and the gene that encodes it is specifically activated during ERS (27). After CIR, HSPA5 dissociates from the three sensors, resulting in their activation, ultimately triggering the ERS-mediated caspase-12/caspase-9/caspase-3 or CHOP apoptosis pathways (11-14). Under ERS, activated PERK specifically phosphorylates eIF2 $\alpha$, inhibits translation of nascent proteins and downregulates the overall level of intracellular protein synthesis (28). In addition, PERK-mediated eIF2 $\alpha$ phosphorylation upregulates ATF4, which activates the pro-apoptotic protein CHOP after ATF4 enters the nucleus (29) (Fig. 6). In the early stage of ERS, phosphorylation of eIF $2 \alpha$ will, to some extent, decrease the load on the ER and promote efficient folding and assembly of proteins, thereby maintaining the steady state of the ER and cellular homeostasis (30). Moreover, when ERS is prolonged, ATF4 transcriptional 
activity is activated in the late phase; this factor helps to initiate apoptosis by driving the expression of the proapoptotic protein CHOP (31) (Fig. 6). Under excessive or long-lasting ERS, the IRE1 $\alpha$ pathway can induce apoptosis by activating JNK and caspase-12, as well as by upregulating transcription of CHOP $(32,33)$. The present study identified that CIRI upregulated the expression levels of components of the PERK and IRE1 pathways, including p-PERK, p-eIF2 $\alpha, p-I R E 1 \alpha$, p-JNK, cleaved caspase-12, CHOP, HSPA5 and the apoptotic protein caspase-3. After OXA treatment, all of these proteins were downregulated, resulting in a lower rate of apoptosis. Thus, OXA exerted a neuroprotective effect following CIRI by inhibiting ERS-mediated apoptosis, which provides a novel method for the treatment of stroke.

The current study has a few limitations. First, the role of $\mathrm{Ca}^{2+}$ in the ERS/caspase-12/caspase-3 apoptosis pathway under OXA treatment was not investigated. The ER is the main $\mathrm{Ca}^{2+}$ reservoir in the cell, and consequently serves a key role in controlling the intracellular $\mathrm{Ca}^{2+}$ concentration. In CIRI, intracellular $\mathrm{Ca}^{2+}$ overload and $\mathrm{ER} \mathrm{Ca}^{2+}$ depletion are both pathophysiological changes worthy of attention, and both cause ERS (34). Under ERS, an increase in intracellular $\mathrm{Ca}^{2+}$ levels results in activation of cytoplasmic calpain and translocation of the ER membrane, activating the caspase-12 precursor and ultimately leading to apoptosis (34). These issues will be addressed in follow-up studies.

In conclusion, the present study demonstrated that OXA exerted a neuroprotective effect against CIRI by inhibiting ERS-mediated apoptosis. Thus, OXA represents a promising basis for a novel treatment strategy for stroke.

\section{Acknowledgements}

Not applicable.

\section{Funding}

This work was supported by grants from National Nature Science Foundation of China (grant nos. 81501018, 81671276 and 31271243), the Natural Science Foundation of Shandong Province (grant no. ZR2018MC005) and the Scientific Research Support Fund for teachers of Jining Medical University (grant nos. JYFC2018JS003 and JYFC2018JS008).

\section{Availability of data and materials}

The datasets used and/or analyzed during the current study are available from the corresponding author on reasonable request.

\section{Authors' contributions}

$\mathrm{CW}$ and JC conceived of and designed the experiments. DX and TK conducted the experiments and wrote the manuscript. RZ, CY and BC performed the data analysis. All authors read and approved the final manuscript.

\section{Ethics approval and consent to participate}

All animal experiments were conducted in accordance with the National Institutes of Health Guide for the Care and Use of Laboratory Animals and approved by the Animal Ethics Committee of Jining Medical University, China (approval no. 2018-JS-001).

\section{Patient consent for publication}

Not applicable.

\section{Competing interests}

The authors declare that they have no competing interests.

\section{References}

1. Jiang G,Li W, Wang D, Shen C,Ji Y and Zheng W: Epidemiological transition and distribution of stroke incidence in Tianjin, China, 1988-2010. Public Health 131: 11-19, 2016.

2. Barthels D and Das H: Current advances in ischemic stroke research and therapies. Biochim Biophys Acta Mol Basis Dis 1866: 165260, 2020.

3. Jiang M, Li J, Peng Q, Liu Y, Liu W, Luo C, Peng J, Li J, Yung KK and Mo Z: Neuroprotective effects of bilobalide on cerebral ischemia and reperfusion injury are associated with inhibition of pro-inflammatory mediator production and down-regulation of JNK1/2 and p38 MAPK activation. J Neuroinflammation 11: 167,2014

4. Chien A and Viñuela F: Analyzing circle of Willis blood flow in ischemic stroke patients through 3D stroke arterial flow estimation. Interv Neuroradiol 23: 427-432, 2017.

5. Bakthavachalam P and Shanmugam PST: Mitochondrial dysfunction - Silent killer in cerebral ischemia. J Neurol Sci 375: 417-423, 2017.

6. Ma XD, Song JN, Zhang M, An JY, Zhao YL and Zhang BF: Advances in research of the neuroprotective mechanisms of cerebral ischemic postconditioning. Int J Neurosci 125: 161-169, 2015.

7. Hu YQ, Chen W, Yan MH, Lai JJ, Tang $\mathrm{N}$ and Wu L: Ischemic preconditioning protects brain from ischemia/reperfusion injury by attenuating endoplasmic reticulum stress-induced apoptosis through PERK pathway. Eur Rev Med Pharmacol Sci 21: 5736-5744, 2017.

8. Wu CX, Liu R, Gao M, Zhao G, Wu S, Wu CF and Du GH: Pinocembrin protects brain against ischemia/reperfusion injury by attenuating endoplasmic reticulum stress induced apoptosis. Neurosci Lett 546: 57-62, 2013.

9. Lv Z, Liu C, Zhai M, Zhang Q, Li J, Zheng F and Peng M: LPS pretreatment attenuates cerebral ischaemia/reperfusion injury by inhibiting inflammation and apoptosis. Cell Physiol Biochem 45: 2246-2256, 2018.

10. Qiu J, Wang X, Wu F, Wan L, Cheng B, Wu Y and Bai B: Low dose of Apelin-36 attenuates ER stress-associated apoptosis in rats with ischemic stroke. Front Neurol 8: 556, 2017.

11. García de la Cadena S and Massieu L: Caspases and their role in inflammation and ischemic neuronal death. Focus on caspase-12. Apoptosis 21: 763-777, 2016.

12. Gorman AM, Healy SJ, Jäger R and Samali A: Stress management at the ER: Regulators of ER stress-induced apoptosis. Pharmacol Ther 134: 306-316, 2012.

13. Maier PJ,Zemoura K, Acuña MA, Yévenes GE, Zeilhofer HU and Benke D: Ischemia-like oxygen and glucose deprivation mediates down-regulation of cell surface $\gamma$-aminobutyric acid B receptors via the endoplasmic reticulum (ER) stress-induced transcription factor CCAAT/enhancer-binding protein (C/EBP)-homologous protein (CHOP). J Biol Chem 289: 12896-12907, 2014.

14. Paschen W and Mengesdorf T: Endoplasmic reticulum stress response and neurodegeneration. Cell Calcium 38: 409-415, 2005.

15. Mattson MP, Duan W, Pedersen WA and Culmsee C: Neurodegenerative disorders and ischemic brain diseases. Apoptosis 6: 69-81, 2001.

16. Kitamura E, Hamada J, Kanazawa N, Yonekura J, Masuda R, Sakai F and Mochizuki H: The effect of orexin-A on the pathological mechanism in the rat focal cerebral ischemia. Neurosci Res 68: 154-157, 2010.

17. Wang CM, Pan YY, Liu MH, Cheng BH, Bai B and Chen J: RNA-seq expression profiling of rat MCAO model following reperfusion Orexin-A. Oncotarget 8: 113066-113081, 2017. 
18. Kong T, Qiu K, Liu M, Cheng B, Pan Y, Yang C, Chen J and Wang C: Orexin-A protects against oxygen-glucose deprivation/ reoxygenation-induced cell damage by inhibiting endoplasmic reticulum stress-mediated apoptosis via the $\mathrm{Gi}$ and PI3K signaling pathways. Cell Signal 62: 109348, 2019.

19. National Research Council (US): Committee for the Update of the Guide for the Care and Use of Laboratory Animals: Guide for the Care and Use of Laboratory Animals. 8th edition. National Academies Press, Washington, DC, 2011.

20. Yuan LB, Dong HL, Zhang HP, Zhao RN, Gong G, Chen XM, Zhang LN and Xiong L: Neuroprotective effect of orexin-A is mediated by an increase of hypoxia-inducible factor-1 activity in rat. Anesthesiology 114: 340-354, 2011.

21. Longa EZ, Weinstein PR, Carlson S and Cummins R: Reversible middle cerebral artery occlusion without craniectomy in rats. Stroke 20: 84-91, 1989.

22. Nakka VP, Gusain A and Raghubir R: Endoplasmic reticulum stress plays critical role in brain damage after cerebral ischemia/ reperfusion in rats. Neurotox Res 17: 189-202, 2010.

23. Wagner DC, Riegelsberger UM, Michalk S, Härtig W, Kranz A and Boltze J: Cleaved caspase-3 expression after experimental stroke exhibits different phenotypes and is predominantly non-apoptotic. Brain Res 1381: 237-242, 2011.

24. Fan J, Lv H, Li J, Che Y, Xu B, Tao Z and Jiang W: Roles of Nrf2/HO-1 and HIF-1 $\alpha /$ VEGF in lung tissue injury and repair following cerebral ischemia/reperfusion injury. J Cell Physiol 234: 7695-7707, 2019.

25. Zhang C, Chen S, Zhang Z, Xu H,Zhang W, Xu D, Lin B and Mei Y: Asiaticoside alleviates cerebral ischemia-reperfusion injury via NOD2/mitogen-activated protein kinase (MAPK)/nuclear factor kappa B (NF- $\mathrm{B}$ ) signaling pathway. Med Sci Monit 26: e920325, 2020

26. El Khashab IH, Abdelsalam RM, Elbrairy AI and Attia AS: Chrysin attenuates global cerebral ischemic reperfusion injury via suppression of oxidative stress, inflammation and apoptosis. Biomed Pharmacother 112: 108619, 2019.
27. DeGracia DJ, Kumar R, Owen CR, Krause GS and White BC: Molecular pathways of protein synthesis inhibition during brain reperfusion: Implications for neuronal survival or death. J Cereb Blood Flow Metab 22: 127-141, 2002.

28. Marciniak SJ, Garcia-Bonilla L, Hu J, Harding HP and Ron D. Activation-dependent substrate recruitment by the eukaryotic translation initiation factor 2 kinase PERK. J Cell Biol 172: 201-209, 2006

29. DeGracia DJ and Montie HL: Cerebral ischemia and the unfolded protein response. J Neurochem 91: 1-8, 2004

30. Scheuner D, Song B, McEwen E, Liu C, Laybutt R, Gillespie P, Saunders T, Bonner-Weir S and Kaufman RJ: Translational control is required for the unfolded protein response and in vivo glucose homeostasis. Mol Cell 7: 1165-1176, 2001.

31. Harding HP, Zhang Y, Bertolotti A, Zeng H and Ron D: Perk is essential for translational regulation and cell survival during the unfolded protein response. Mol Cell 5: 897-904, 2000.

32. Gupta S, Deepti A, Deegan S, Lisbona F, Hetz C and Samali A: HSP72 protects cells from ER stress-induced apoptosis via enhancement of IRE1alpha-XBP1 signaling through a physical interaction. PLoS Biol 8: e1000410, 2010.

33. Roberson EC, Tully JE, Guala AS, Reiss JN, Godburn KE, Pociask DA, Alcorn JF, Riches DW, Dienz O, JanssenHeininger YM, et al: Influenza induces endoplasmic reticulum stress, caspase-12-dependent apoptosis, and c-Jun N-terminal kinase-mediated transforming growth factor- $\beta$ release in lung epithelial cells. Am J Respir Cell Mol Biol 46: 573-581, 2012.

34. Verkhratsky A and Petersen $\mathrm{OH}$ : The endoplasmic reticulum as an integrating signalling organelle: From neuronal signalling to neuronal death. Eur J Pharmacol 447: 141-154, 2002.

This work is licensed under a Creative Commons

Attribution-NonCommercial-NoDerivatives 4.0 International (CC BY-NC-ND 4.0) License. 